Provided for non-commercial research and education use. Not for reproduction, distribution or commercial use.

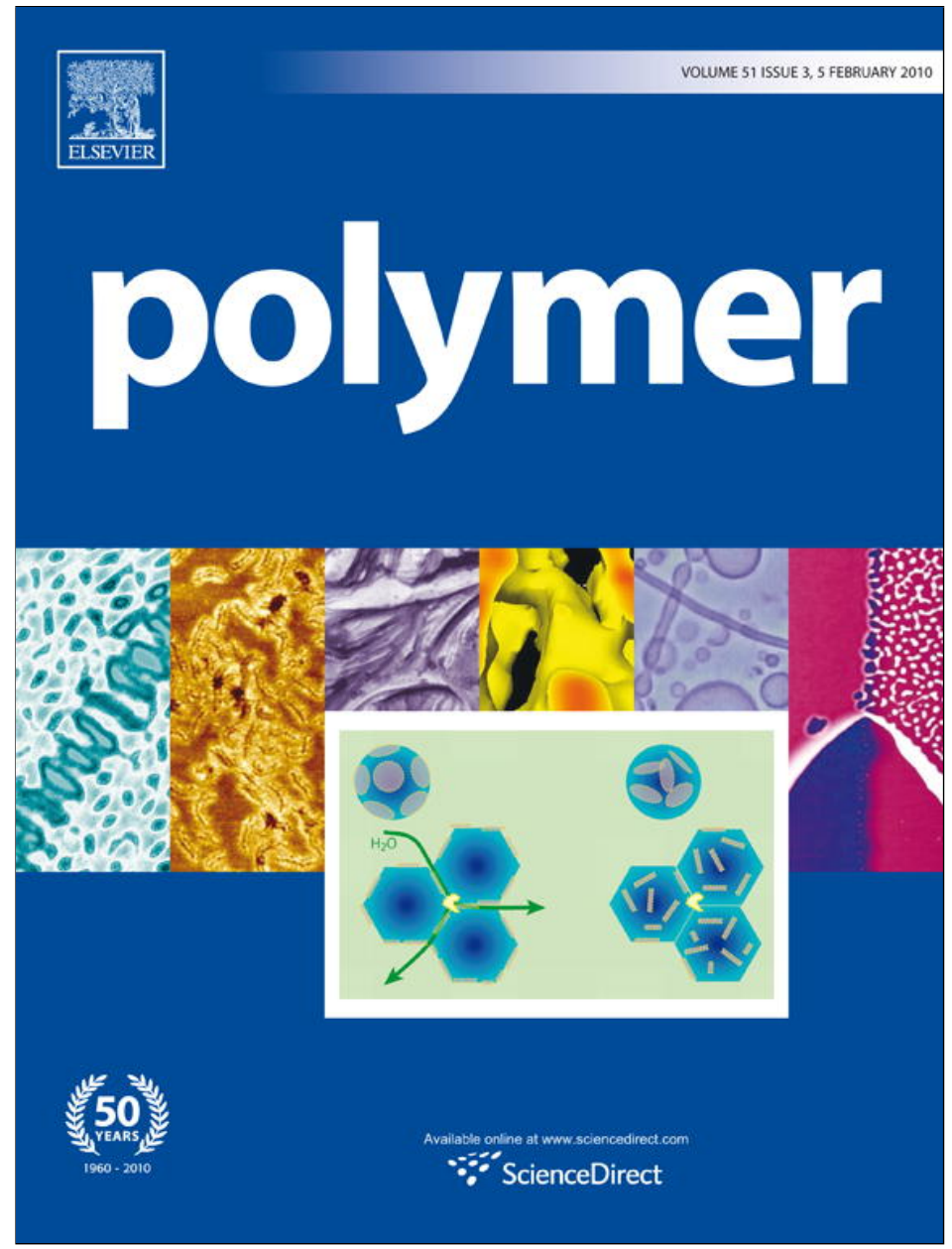

This article appeared in a journal published by Elsevier. The attached copy is furnished to the author for internal non-commercial research and education use, including for instruction at the authors institution and sharing with colleagues.

Other uses, including reproduction and distribution, or selling or licensing copies, or posting to personal, institutional or third party websites are prohibited.

In most cases authors are permitted to post their version of the article (e.g. in Word or Tex form) to their personal website or institutional repository. Authors requiring further information regarding Elsevier's archiving and manuscript policies are encouraged to visit:

http://www.elsevier.com/copyright 


\title{
Formation of silicone elastomer networks films with gradients in modulus
}

\author{
Julie A. Crowe-Willoughby ${ }^{1}$, Katherine L. Weiger, Ali E. Özçam, Jan Genzer* \\ Department of Chemical \& Biomolecular Engineering, North Carolina State University, Raleigh, NC 27695-7905, USA
}

\section{A R T I C L E I N F O}

\section{Article history:}

Received 22 July 2009

Received in revised form

15 November 2009

Accepted 21 November 2009

Available online 21 December 2009

\section{Keywords:}

Silicone elastomer

Gradient

Modulus

\begin{abstract}
A B S T R A C T
We describe the formation of soft material substrates with position-dependent modulus. Two strategies have been employed in the preparation of such materials. In the first method, we create modulus gradient structures by inter-diffusing two (or more) formulations of silicone elastomers (SE), comprising mixtures of poly(dimethylsiloxane) (PDMS) and poly(vinylmethylsiloxane) (PVMS) with varying molecular weight and content of PDMS, and cross-link them thermally in order to form SE networks. With this method, the resultant substrates exhibit shallow modulus gradients that extend over a few centimeters in length. The second technique is based on employing ultraviolet (UV)-based cross-linking of mercapto-terminated PVMS chains across transparency lithographic masks. We demonstrate that the shape and "sharpness" of the modulus on the substrate depends solely on the position-dependent transparency of the mask to the UV light. As a part of the study we provide detailed material characterization of networks formed by the PDMS-PVMS and PVMS-SH chains.
\end{abstract}

(ㄷ) 2009 Elsevier Ltd. All rights reserved.

\section{Introduction}

One of the major tenets of materials science involves developing reliable methods of tuning the characteristics of surfaces and interfaces in materials. While just about any material property can, in principle, be altered, the most important ones that impact the performance of both hard and soft material surfaces involve variation of their surface energy, surface roughness (i.e., topography) and film mechanical properties (i.e., modulus). Multiple methods that facilitate tuning the surface energy and (to some extent) surface roughness have been developed and described in many previous monographs. In contrast, formation of thin polymer layers with tunable mechanical characteristics has received much less attention. Yet, the latter property has long been known to govern some key characteristics of materials, including mechanical stability [1] and wear [2]. In addition, modulus has been utilized in controlling surface topography by capitalizing on material instabilities, such as wrinkling/buckling [3]. Recent studies have also revealed that mechanical properties of substrates govern interfacial partitioning of certain biomolecules. For instance, Brady and Singer

\footnotetext{
* Corresponding author. Tel.: +1 919515 2069; fax: +1 9195153465.

E-mail addresses: julie.willoughby@gmail.com (J.A. Crowe-Willoughby), Jan_ Genzer@ncsu.edu (J. Genzer).

1 Present address: MeadWestvaco, Center for Packaging Innovation, 1021 Main Campus Drive, Raleigh, NC 27606.
}

reported that relative bioadhesion on various polymeric surfaces scales with $\left(\gamma_{c} E\right)^{1 / 2}$, where $\gamma_{c}$ and E are the critical surface energy and the elastic modulus, respectively [4,5]. The role of material stiffness on bioadhesion is not completely new, however. Cell biologists have known for some time that when cultured on solid substrates, e.g., tissue-culture polystyrene dishes, cells proliferate readily and rapidly relative to situations involving more compliant materials [6]. They also established that surface chemistry and materials mechanics act in concert in regulating biological functions. Indeed, recent observations have indicated that cells may alter their biological functions, i.e., signaling pathways, based on the substrate stiffness [7].

While surface chemistry and topology have been probed extensively for their effect on such responses as cellular adhesion and proliferation, studies are beginning to emerge regarding the effect of substrate modulus [8,9]. In order to achieve timely comprehensive experimental design, there is a need to create novel substrates with tunable mechanical response at the micro and meso-scale levels with length scales ranging from individual cells up to whole tissues $[10,11]$. Despite the vast amount of research that has been targeted at understanding cellular response to its host scaffold, the choice of material and extrapolation of findings from one cell/material system to another system has proven difficult, indicating that establishing general relationships between substrate compliance and cell behavior cannot be considered independently of the material and cell type. Over the past few years, the effect of substrate compliance on cell motility, adsorption and proliferation has been investigated systematically by means of 
substrates bearing a gradient in mechanical modulus [12]. Polyacrylamide (PAAm) has been an attractive polymer for these studies because the acrylamide (AAm) monomer can be photo-cured into a network and subsequently chemically modified (through the pendent $-\mathrm{OCNH}_{2}$ substituents) with cell-adhesive ligands. In their pioneering work of Wang and co-workers utilized PAAm-based networks to demonstrate cell motion driven by the substrate modulus, so-called durotaxis. In their experiments, NIH 3T3 mouse cells migrated from the softer region (Young's modulus $\approx 14 \mathrm{kPa}$ ) to the stiffer regions (Young's modulus $\approx 30 \mathrm{kPa}$ ). Wong and coworkers later extended those studies by fabricating PAAm-based gradients with tuned length scales and elastic moduli and following durotaxis of vascular smooth muscle cells [8-10]. While insightful in providing the first glimpse at the effect of substrate mechanical properties on the cell partitioning, the PAAm-based materials are not ideally suited for such studies. First, AAm may be toxic when applied in vivo. Second, PAAm, like any other hydrogel, swells in water thus modifying greatly the modulus of the networks. A silicon elastomer (SE)-based substrate as an engineering scaffold may be more desirable material for cell-surface study than a PAAm support due to their physiologically relevant mechanical properties and applicability as FDA-approved biomaterials [13]. However, there are complications with utilizing PDMS, the most common SE, as a substrate; these arise due to inherent resistance of PDMS to chemical modification with cell-adhesive ligands [10]. This latter problem can be circumvented by utilizing functional SEs, such as poly(vinylmethylsiloxane) (PVMS) or poly(hydromethylsiloxane) (PHMS), as building blocks for the networks. To that end, the presence of the vinyl functionality in PVMS and the hydride group in PHMS can be employed not only to incorporate these SEs into the network but also provide abundant density of reactive sites for attaching biological ligands.

In this work we outline two methodologies for fabricating substrates comprising a gradient in surface modulus gradients by means of functional SE materials. In the first strategy we achieve the gradient by inter-diffusing SEs mixtures of PDMS and PVMS with different molecular weights and compositions across a substrate. Here the spatial extent of the resulting gradient substrate is dictated solely by the diffusivity of the individual SE mixture counterparts. The network formation is achieved by heating. In the second scenario, we offer a more general strategy by utilizing end-functional mercapto-terminated PVMS coatings and control the degree of cross-linking by exposing the materials to UV light. By spatially controlling the distribution of the UV light intensity during the UV-curing step via transparency masks we prepare material gradients in which the spatial distribution of the modulus is dictated exclusively by the characteristics of the transparency mask. Results of our studies utilizing these modulus gradient substrates will be described in a separate publication [14].

\section{Materials and methods}

\subsection{Preparation of PDMS-PVMS networks}

The SE networks were prepared by reacting hydride-terminated PDMS (Gelest) across the PVMS backbone and cured via standard cure by means of the platinum catalyst [15]; the synthesis of PVMS was described elsewhere [16]. A typical formulation for a 11-75 PDMS-PVMS network ( NPDMS $-V_{\text {PVMS }}$, where $\mathrm{N}_{\text {PDMS }}$ denotes the degree of polymerization of PDMS-H and $\mathrm{V}_{\mathrm{PVMS}}$ denotes the number of vinyl groups on the PVMS chain to be reacted) was a follows: $20 \mathrm{~g}$ of PVMS fluid $\left(M_{\mathrm{w}}=45 \mathrm{kDa}\right), 75 \mu \mathrm{l}$ of Pt (0) from Aldrich, $50 \%$ in poly(vinylmethylsiloxane), and $6 \mathrm{~g}$ of hydrideterminated PDMS (Gelest H11-Fluid). The order of mixing is important to prevent premature gelation between the catalyst and the hydride-terminated PDMS. The best procedure is to thoroughly mix the PVMS fluid with catalyst prior to the addition of the H11. A lab mixer was used for uniform distribution of the components necessitating de-airing via a vacuum chamber. After the mixture was poured into the desired container, it was placed in a $70^{\circ} \mathrm{C}$ oven for 2 days and then at room temperature for at least 5 days prior to use.

\subsection{Preparation modulus gradients by inter-diffusion}

The mixtures of varying composition were prepared as described above and poured into the desired container in equal increments. The most successful gradient was generated in the large $30 \times 30 \mathrm{~cm}^{2}$ Petri dish but formulations on a $10 \times 25 \mathrm{~mm}$ scale were also prepared (data not shown). Once the formulations appeared to have diffused "evenly", they were cured as described above.

\subsection{Preparation of PVMS-SH polymers}

This synthesis was performed under vacuum to obtain very high molecular weight materials with the option of adding endblockers to the formulation enabling various terminus functionality of the resulting siloxane fluid. Adjusting the process to produce a mercapto-terminated PVMS (PVMS-SH) fluid did not require the use of vacuum as we were not trying to build the molecular weight of the starting PVMS-OH fluid. Ideally, the mercaptoalkoxysilane should be monofunctional in order to prevent cross-linking and gelation during the synthesis. This monoalkoxysilane was not available commercially, however. Since its synthesis was not straightforward, 3-mercaptopropylmethyl dimethoxysilane was employed as the end-blocker [17]. In order to promote the condensation reaction between the primary alkoxysilane and the -OH terminal moieties of PVMS, the reaction was heated under reflux to $65{ }^{\circ} \mathrm{C}$ with $0.1 \mathrm{wt} \%$ barium hydroxide. The reaction completion was determined with FTIR, which monitored the disappearance of the hydroxy peaks of the siloxane due to the capping with the 3-mercaptodimethoxysilane (cf. Fig. S2 in the Supplementary information). Because the degree of polymerization of PVMS-OH (N $\mathrm{N}_{\mathrm{PVMS}-\mathrm{OH}}$ ) increased from 15 to 300 , the dimethoxysilane was added in $2-4$ fold excess formulated at 2:1 mol of end-blocker per mole of PVMS-OH to limit step-growth polymerization. With high molecular weight PVMS-OH ( $\mathrm{N}_{\mathrm{PVMS}-\mathrm{OH}}=300$ ), branching occurring during the endcapping reaction could result in an uncontrollable rate of reaction. In order to limit the molecular weight increase, the catalyst was added after the reaction temperature reached $60{ }^{\circ} \mathrm{C}$. The reaction was held at $60{ }^{\circ} \mathrm{C}$ for $2.5 \mathrm{~h}$. After the end of the reaction time, heating was terminated and the barium catalyst was removed from the resultant product by filtering through a cake of diamateous earth (Celite 545 from Aldrich) twice, which effectively terminated the reaction. The product was a clear viscous liquid that was further purified by precipitation into methanol in order to remove any residual 3-mercaptodimethoxysilane. We note that due to the di-functional nature of the silane end-capper, we cannot exclude the possibility that some condensation of the dangling unreacted methoxysilane group can occur leading to a free $-\mathrm{OH}$. This may, in turn, undergo further condensation. Given the relative stability of the methoxy groups, if such condensation indeed takes place, it should happen during the reflux heating rather than at room temperature. The modulus of the final network is thus not expected to be altered substantially provided it is not exposed to high temperatures.

As the hydroxy peaks in the precursor PVMS-OH ( $\mathrm{N}_{\mathrm{PVMS}-\mathrm{OH}}=$ 300) were not apparent with FTIR and $-\mathrm{SiOCH}_{2}$ - and $-\mathrm{SH}$ stretching vibrations exhibit weak frequency signals $\left(2840 \mathrm{~cm}^{-1}\right.$ and 2560 
$\mathrm{cm}^{-1}$, respectively), we confirmed that the reaction took place with ${ }^{13} \mathrm{C}$ and ${ }^{1} \mathrm{H}$ NMR (cf. Fig. S3 and Fig. S4 in the Supplementary information). Upon inspection of the spectra for both PVMS-OH $\left(\mathrm{N}_{\mathrm{PVMS}-\mathrm{OH}}=15\right.$, PJ Fluid $)$ and PVMS-OH $\left(\mathrm{N}_{\mathrm{PVMS}-\mathrm{OH}}=300\right)$ in comparison to PVMS-SH, there are obvious differences in the 0.5$3.5 \mathrm{ppm}$ region for ${ }^{1} \mathrm{H}$ and in $12-53 \mathrm{ppm}$ region for ${ }^{13} \mathrm{C}$ indicating chemical shifts due to the mercapto-termination $[18,19]$. Specifically, in Fig. S4 the ${ }^{1} \mathrm{H}$ chemical shifts for PVMS-SH that are absent for PVMS (cf. Fig. S3) include: i) the protons on the methoxy group occurs $\approx 3.5 \mathrm{ppm}$, ii) the proton on the $-\mathrm{S}$ - atom occurs around $1.3-$ $1.6 \mathrm{ppm}$, and iii) the propyl spacer between sulfur and silicon has proton chemical shifts ranging from 0.9 to $2.5 \mathrm{ppm}$. The significant peaks in the ${ }^{13} \mathrm{C}$ NMR spectra occur at $50 \mathrm{ppm}$ for the carbon from the methoxy group and between 15 and $35 \mathrm{ppm}$ for the carbons in the propyl spacer.

After synthesizing PVMS-SH with both short chain PVMS-OH $\left(\mathrm{N}_{\mathrm{PVMS}-\mathrm{OH}}=15\right)$ and PVMS-OH $\left(\mathrm{N}_{\mathrm{PVMS}-\mathrm{OH}}=300\right)$, their molecular weight was determined with size exclusion chromatography (SEC). Fig. S5 in the Supplementary information illustrates the SEC differential refractive index (DRI) detector traces for the nominal $\mathrm{N}_{\text {PVMS-OH }}=300$ PVMS-OH and the resultant PVMS-SH (B). Note that the weight-average molecular weight $\left(M_{\mathrm{W}}\right)$ increased from 28 to 57 $\mathrm{kDa}$ after the end-capping condensation reaction indicating either branching from the secondary alkoxysilane group or chain extension with PVMS-OH step-growth condensation. The DRI traces show 3 peaks for each sample. The first peak represents the linear PVMS polymer, the second peak corresponds to either vinylmethyl cyclics or low molecular weight linear species, and the third peak is associated with the solvent from the sample solution. Consistent with the increase in molecular weight is the decrease in short chain/cyclic siloxanes in the PVMS-SH trace as well as a broadening of the main peak. It is interesting to note that even when starting out with low molecular weight ( $\left.\mathrm{N}_{\mathrm{PVMS}-\mathrm{OH}}=15\right) \mathrm{PVMS}-\mathrm{OH}$, the molecular weight increases from $\approx 1.5$ to $\approx 42 \mathrm{kDa}$. The concentration of the condensation catalyst for the N $\mathrm{PVMS}_{\mathrm{OH}}=15$ PVMS$\mathrm{OH}$ run was one third the amount used for the high molecular weight PVMS-OH illustrating the differences in reaction rates for the different viscosity fluids. As the mixing apparatus was the same for both runs, the catalyst amount was a means to overcome the mass transfer limitations of the low molecular weight end-blocker species throughout the more viscous PVMS-OH fluid. The reaction starting with PVMS-OH (NPVMS-OH $=15$ ) demonstrates that the synthesis of PVMS-SH can be a one-step reaction of end-capping and step-growth condensation occurring simultaneously. Starting with the higher molecular weight material was an attempt to control the amount of branching and prevent the reaction between the secondary methoxysilane and the hydroxy group from occurring. Inspection of the SEC traces in Fig. S5 reveals that while the resultant product with the higher molecular weight PVMS-OH material produced a narrower molecular weight distribution of final polymer, a low molecular weight tail was present in both cases.

\subsection{Wettability measurements}

Static contact angle measurements were performed via the sessile drop technique with deionized water (DIW, resistance $>15$ $\mathrm{M} \Omega \mathrm{cm}$ ) using a Ramé-Hart contact angle goniometer (Model 10000) equipped with a CCD camera. Images were analyzed with the Ramé-Hart Imaging 2001 software. To measure the advancing and receding contact angles, a probe droplet was added $(8 \mu \mathrm{L}$, DIW advancing) or removed ( $4 \mu \mathrm{L}$ DIW, receding) and analyzed. Contact angles were measured on 3-5 independent spots on each sample and the results were averaged. The dynamic contact angle (DCA) measurements were carried out on Cahn dynamic contact angle analyzer. The contact angle hysteresis, defined as the difference between the advancing and receding contact angles of DIW, was evaluated as a measure of the chemical and structural homogeneity of the surface probed.

\subsection{Dynamic rheology and dynamical mechanical thermal analysis (DMTA)}

Dynamic frequency sweeps were performed under isothermal conditions from 0.05 to $100 \mathrm{rad} / \mathrm{s}$ on a Rheometrics Advanced Rheometric Expansion System (ARES) with a defined strain within the linear viscoelastic regime using a parallel plate geometry. Wall slip was minimized my applying a normal force for optimum plate contact producing repeatable results. DMTA was performed using a TA Instrument Q800 operating in a single-cantilever geometry. The dried polymers films were cut into approximately $25 \times 10 \times 1.5$ $\mathrm{mm}^{3}$ rectangles. Storage modulus $\left(\mathrm{E}^{\prime}\right)$, loss modulus $\left(E^{\prime \prime}\right)$, and loss tangent $(\tan \delta)$ were recorded at a heating rate of $3{ }^{\circ} \mathrm{C} / \mathrm{min}$ from -150 to $130{ }^{\circ} \mathrm{C}$ and at a test frequency of $1 \mathrm{~Hz}$. The amplitude was set to be within the linear viscoelastic regime.

\subsection{Fourier transform infrared (FTIR) spectroscopy}

Fourier transform infrared spectroscopy in the attenuated total reflection mode (FTIR-ATR) was employed to characterize chemical modification of PVMS-OH fluids. All spectra were collected using a Bio-Rad/Digilab FTS-3000 Fourier transform infrared (FT-IR) spectrometer using a mounted crystalline Germanium, attenuated total internal reflection (ATR) sampling attachment (Pike Technologies inc., MIRacle ${ }^{\mathrm{TM}}$ Single Reflection ATR) with a normal spectral response of $650-5500 \mathrm{~cm}^{-1}$. The infrared light was focused onto the photodiode of a liquid nitrogen-cooled, wide band mercurycadmium-telluride (MCT) detector with a normal spectral response of $650-7000 \mathrm{~cm}^{-1}$. The spectrometer and attachment were purged with dry compressed air in order to remove signals originating from atmospheric water and $\mathrm{CO}_{2}$. For each sample, 64 scans (10 repeats per sample) were collected using Ge-crystal detector with a resolution of $2 \mathrm{~cm}^{-1}$ under constant nitrogen flux to eliminate the effect of water vapor on the collected data. The data were analyzed using BioRad-IR software.

\subsection{Nanoidentation experiments}

Testing of the mechanical properties of modified and unmodified PVMS networks were performed by using Hysitron Triboindenter. The instrument was equipped with quasi-static and dynamic mode of operation, force and displacement controlled (feedback) and an integrated AFM head. Indentations were performed at room temperature in the acoustic enclosure of the Triboindenter. 2 different tips were used to perform indentations, a $150 \mathrm{~nm}$ Berkovic and a $46 \mu \mathrm{m}$ conical diamond tip, the calibration of the diamond tips were performed on standard fused quartz. Force-displacements curves of indents were analyzed by using Oliver-Pharr method by using the software TriboScan supplied by Hysitron. Only the results of displacement controlled quasi-static indentations were reported. The reduced modulus is calculated using the equation:

$$
\begin{aligned}
& E_{r}=\frac{S}{2} \frac{\sqrt{\pi}}{\sqrt{A}} \\
& \frac{1}{E_{r}}=\left(\frac{1-v^{2}}{E}\right)_{\text {sample }}+\left(\frac{1-v^{2}}{E}\right)_{\text {indenter }}
\end{aligned}
$$


where $(S)$ is the stiffness of the unloading curve, $(A)$ is the projected contact area, and $\nu$ is Poisson's ratio (taken to be $\approx 0.5$, as for ideal elastomers). The initial unloading contact stiffness (the slope of the initial portion of the unloading curve) is defined as:

$S=\frac{\mathrm{d} P}{\mathrm{~d} h}$

For a standard diamond indentor probe, $E_{\text {indenter }}$ is $1140 \mathrm{GPa}$ and

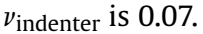

The samples were cured on glass slides and glass slides were mounted on the magnetic plate by attaching metal AFM discs under glass slides with super glue. A series of indentations were performed on each sample by considering the minimum distance not to effect the neighboring indentations and the size of the each sample. In separate experiments standard deviation of the modulus were calculated on homogenous modulus samples and they are found to be $\approx 3 \%$ of the modulus values. In addition, the indented surface was scanned with the integrated AFM in non-contact mode and no visible deformation or cut on the network was observed. The same test conditions were used for each sample and the test parameters for displacement controlled indentations are as follows: $500 \mathrm{~nm} / \mathrm{s}$ loading, $5 \mathrm{~s}$ of hold period and $500 \mathrm{~nm} / \mathrm{s}$ unloading.

\subsection{NMR spectroscopy}

The pulsed field NMR experiments were performed on a Bruker AVANCE $500 \mathrm{MHz}$ Spectrometer (1996) with Oxford Narrow Bore Magnet (1989), SGI INDY Host Workstation, XWINNMR Software version. Instrument is equipped with three Frequency Channels with Wave Form Memory and Amplitude Shaping Unit, with three Channel Gradient Control Unit (GRASP III), variable Temperature Unit, Pre-Cooling and Temperature Stabilization Unit. $5 \mathrm{~mm}$ ID 1H/ BB $\left({ }^{109} \mathrm{Ag}^{31} \mathrm{P}\right)$ Triple-Axis Gradient Probe (ID500-5EB, Nalorac Cryogenic Corp.) has been used for all measurements. The NMR probe was tuned to C-13 frequency, which is $125.75 \mathrm{MHz}$ in the 500 $\mathrm{MHz}$ spectrometer $\left({ }^{1} \mathrm{H}\right.$ frequency $-500.128 \mathrm{MHz}$ ). The NMR probe was tuned to $\mathrm{Si}-29$ frequency, which is $99.36 \mathrm{MHz}$ in the $500 \mathrm{MHz}$ spectrometer $\left({ }^{1} \mathrm{H}\right.$ frequency $\left.-500.128 \mathrm{MHz}\right)$. A combination of homonuclear ${ }^{1} \mathrm{H},{ }^{13} \mathrm{C}$ and ${ }^{29} \mathrm{Si}$ methods were applied to study the structure of polymer. Chromium(III) acetyl acetonate (Aldrich) was used as an efficient relaxation reagent for ${ }^{29} \mathrm{Si}$ NMR studies. ${ }^{13} \mathrm{C}$ (1D) and ${ }^{1} \mathrm{H}$ spectrum were also run with proton decoupling for confirmation of the peak assignments.

The NMR samples were prepared by dissolving $0.2 \mathrm{~g}$ of each sample in approximately $0.5 \mathrm{~g}$ of $\mathrm{CDCl}_{3}$ then transferring the solution to a 5-mm NMR tube for analysis. Tubes were carefully washed and dried for $24 \mathrm{~h}$ in an oven and purged with nitrogen before being capped for storage. Samples for ${ }^{29} \mathrm{Si}$ were prepared with $1 \%$ of relaxation agent to shorten the $T_{1}$ relaxation time [19]. All NMR spectra were acquired at $298 \mathrm{~K}$. Tetramethylsilane was used as an internal standard. Data were processed with Bruker software XWINMR 3.6 and standard processing parameters.

\section{Results and discussion}

\subsection{Substrates with gradient in modulus based on inter-diffusion of SES}

The SE networks for this study were prepared by reacting hydride-terminated PDMS across the PVMS backbone and cured via the platinum catalyst, as shown in Scheme 1. The modulus of the PDMS-PVMS network can be adjusted in two ways: i) by varying the degree of polymerization of the PDMS linker (NPDMS) and/or ii) by varying the number of vinyl groups on the PVMS chain to be

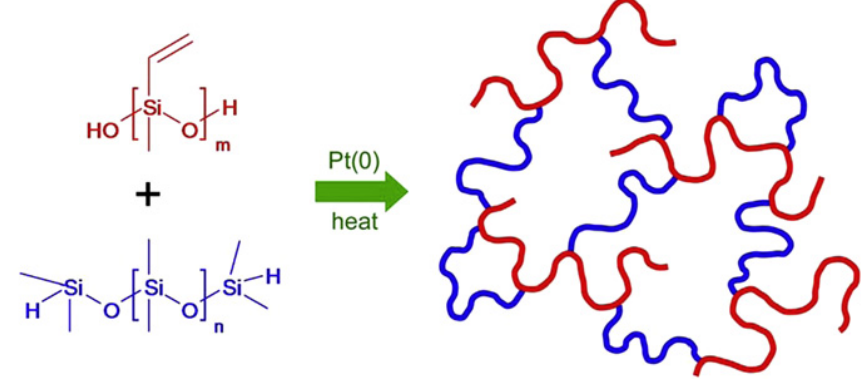

Scheme 1. Schematic of PVMS/PDMS cross-linking mechanism. The amount and molecular weight of the hydride-terminated PDMS as well as the number of vinyl groups targeted for the cross-coupling reaction determine the degree of cross-linking.

reacted $\left(\mathrm{V}_{\mathrm{PVMS}}\right)$. In our studies we used $\mathrm{N}_{\mathrm{PDMS}}$ ranging from 11 to 78 repeat units. Since the average molecular weight of PVMS was $\approx 40$ $\mathrm{kDa}$ (see Experimental section), the maximum number of vinyls per mole of PVMS was $\approx 465$. Targeting no more than 300 vinyl groups/ mole of polymer for reaction provided a sufficiently high number of vinyl reactive sites on the PVMS backbone for subsequent chemical functionalization with biological ligands. In the text we refer to different PDMS-PVMS formulations as NPDMS $-V_{\text {PVMS }}$; for instance, the SE network denoted as 11-300 was made of PDMS having N NDMS $=11$ and $\mathrm{V}_{\mathrm{PVMS}}=300$. We note that a big advantage to this chemistry, particularly with respect to the cell culture studies, is that it avoids the toxicity of the tin catalyst used in alkoxy-cure cross-linking.

We anticipated that the SE network modulus would decrease by increasing $\mathrm{N}_{\text {PDMS }}$ and/or decreasing $\mathrm{V}_{\text {PVMS. }}$ In Fig. 1 we plot the storage modulus $\left(G^{\prime}\right)$ data from a few PDMS-PVMS formulations confirming the expected trend; namely, $G^{\prime}$ decreases with increasing $\mathrm{N}_{\text {PDMS }}$ and/or decreasing $\mathrm{V}_{\text {PVMS. }}$ In several instances, we detected a few variations to this rule. For instance, in the $11-\mathrm{V}_{\mathrm{PVMS}}$ series we saw a consistent increase in $G^{\prime}$ associated with increasing $\mathrm{V}_{\text {PVMS }}$ from 75 to 175 while further increase in $V_{\text {PVMS }}$ to 200 , produced $15-200$ networks whose $G^{\prime}(\approx 300 \mathrm{kPa}$ ) was lower than that of $15-175$ ( $\approx 400 \mathrm{kPa})$. Measurements carried out on duplicate

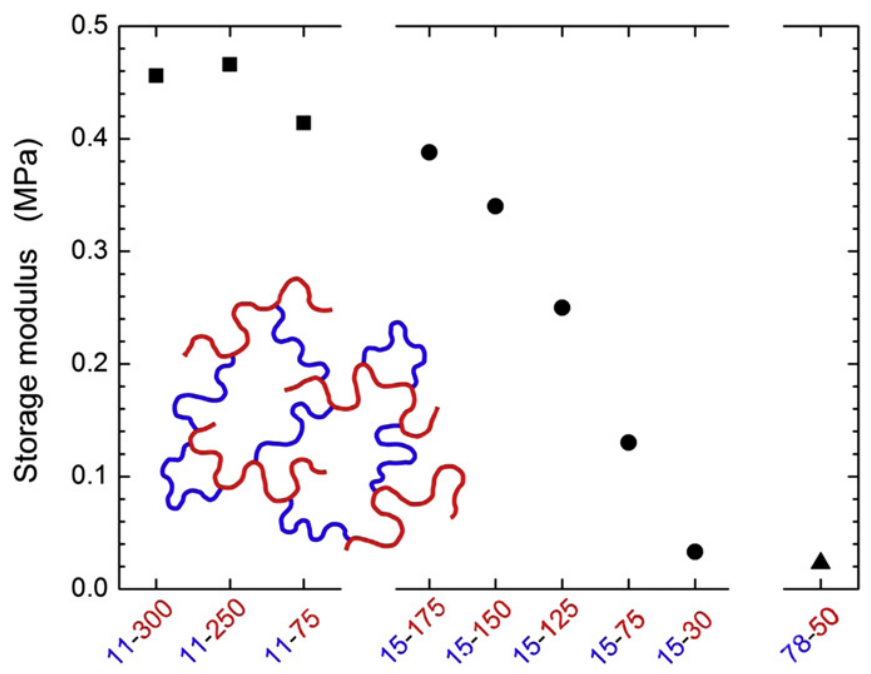

Fig. 1. Formulations for the PVMS/PDMS network blends. The nomenclature is $\mathrm{N}_{\text {PDMS }}-\mathrm{V}_{\mathrm{PVMS}}$, where NPDMS denotes the degree of polymerization of the hydrideterminated PDMS (PDMS-H) fluid and $\mathrm{V}_{\mathrm{PVMS}}$ is the number of vinyls targeted for crosslinking. The degree of polymerization for PDMS-H was $11(\boldsymbol{\bullet}), 15(\bullet)$, and $78(\boldsymbol{\Delta})$. The average degree of polymerization of PVMS (= number of vinyl available for crosslinking) was $\approx 465$. 
batches of 15-200 confirmed consistent results. It is likely that low molecular weight species (PDMS in this case) diffuse to the polymer-air interface of these unextracted samples because of reduced miscibility between PDMS and PVMS and the lower surface energy of PDMS (relative to that of PVMS). It is also possible that a maximum loading had been reached in the incorporated crosslinker molecules and the "extra" PDMS- $\mathrm{H}$ acted as a plasticizer. While this observation constitutes an interesting problem of its own merit, it is beyond the scope of this work to explore it further. Therefore in the preparation of samples comprising modulus gradients, we only used those PDMS-PVMS formulations that followed the expected trends ( $c f$. Fig. 1). Using samples in the $11-V_{\text {PVMS }}$ and $15-\mathrm{V}_{\mathrm{PVM}}$ sets we thus confirmed that the storage modulus followed the expected trends; it decreased with increasing $\mathrm{N}_{\text {PDMS }}$

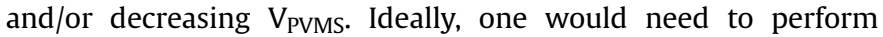
experiments with a broader range of NPDMs. Unfortunately, no hydrogen-terminated PDMS materials are available commercially that would have $15<\mathrm{N}_{\text {PDMS }}<78$. We have tested $\mathrm{N}_{\text {PDMS }}=78$ and prepared 78-50, whose modulus confirmed the previouslydescribed trends in the storage modulus; our attempts to prepare PDMS-PVMS blends with a higher PDMS loading (thus higher $\mathrm{V}_{\mathrm{PVMS}}$ ) led to phase separation. Because of the sample heterogeneity, we have not tested their mechanical properties.

More information about the rheological characteristics of the PDMS-PVMS formulations was obtained from dynamical mechanical thermal analysis (DMTA), which was conducted on two samples, 11-250 and 11-75. The specimens were tested as described in the Experimental section, utilizing the temperature ramp function at $1 \mathrm{~Hz}$ and set amplitude of $40 \mu \mathrm{m}$. The storage ( $\left.E^{\prime}, \mathbf{a}\right)$, loss modulus $\left(E^{\prime}, \square\right)$ (left ordinate) and $\tan \delta(\diamond)$ for 11-250 and 11-75 are shown as a function of temperature in Fig. 2a and Fig. 2b, respectively. The largest step change in modulus for 11-250 (cf. Fig. 2a) is around $150 \mathrm{~K}$, typical of the glass transition temperature $\left(T_{\mathrm{g}}\right)$ of PDMS and PVMS. This large step transition in modulus for 1175 (cf. Fig. $2 \mathrm{~b}$ ) occurs at $145 \mathrm{~K}$. Based on $\tan \delta, T_{\mathrm{g}}$ is $\approx 150$ and $\approx 160$ $\mathrm{K}$ for the 11-75 and 11-250 formulations, respectively. There is a $\beta$ transition apparent in the loss modulus for 11-250 ( $c f$. Fig. 2a) at $141 \mathrm{~K}$ that is absent in the data for PVMS and model PDMS networks ( $c f$. Fig. S1 in the Supplementary information) and not present in the 11-75 data (cf. Fig. 2b). In addition, as the concentration of PDMS in $11-250$ is higher than that of than PVMS $(\approx 60$ weight\% of PDMS-H), the cold crystallization behavior observed with the model homopolymer network was also expected to occur in the 11-250 specimen. This did not occur, however. We note that while the networks may have a similar molecular make-up, their cross-linking mechanism is much different. With the model PDMS and PVMS networks the cross-linking occurred at the end-groups of the macromolecule, whereas the PDMS-PVMS networks formed by cross-linking along the PVMS backbone. The main backbone in all three networks had a similar molecular weight. Thus, while the molecular weight between cross-links $\left(M_{\mathrm{C}}\right)$ for the end-linked networks was identical to that of the backbone, the 11-250 specimen exhibited a short and non-uniform $M_{\mathrm{C}}$ throughout the network. This may explain the observed differences of the loss modulus' secondary temperature transitions.

From the data in Fig. 2 there is a large change in the storage modulus $\left(\Delta E^{\prime}\right)$ associated with the transition from the glassy to the rubbery state between the $11-250\left(\Delta E^{\prime} \approx 10^{3} \mathrm{MPa}\right)$ and 11-75 $\left(\Delta E^{\prime} \approx 7 \times 10^{3} \mathrm{MPa}\right)$ networks. While in the rubbery state, the $11-250$ mixture forms a constrained network with a significant degree of cross-linking, the glassy state storage modulus for $11-250$ is almost an order of magnitude smaller less than that of $11-75$ (i.e., $10^{3} \mathrm{vs}$. $10^{4} \mathrm{MPa}$ ). We currently cannot provide a plausible explanation for the difference in $E^{\prime}$ at temperatures below $T_{\mathrm{g}}$, between the two networks. Another anomaly in the DMTA data of the two networks
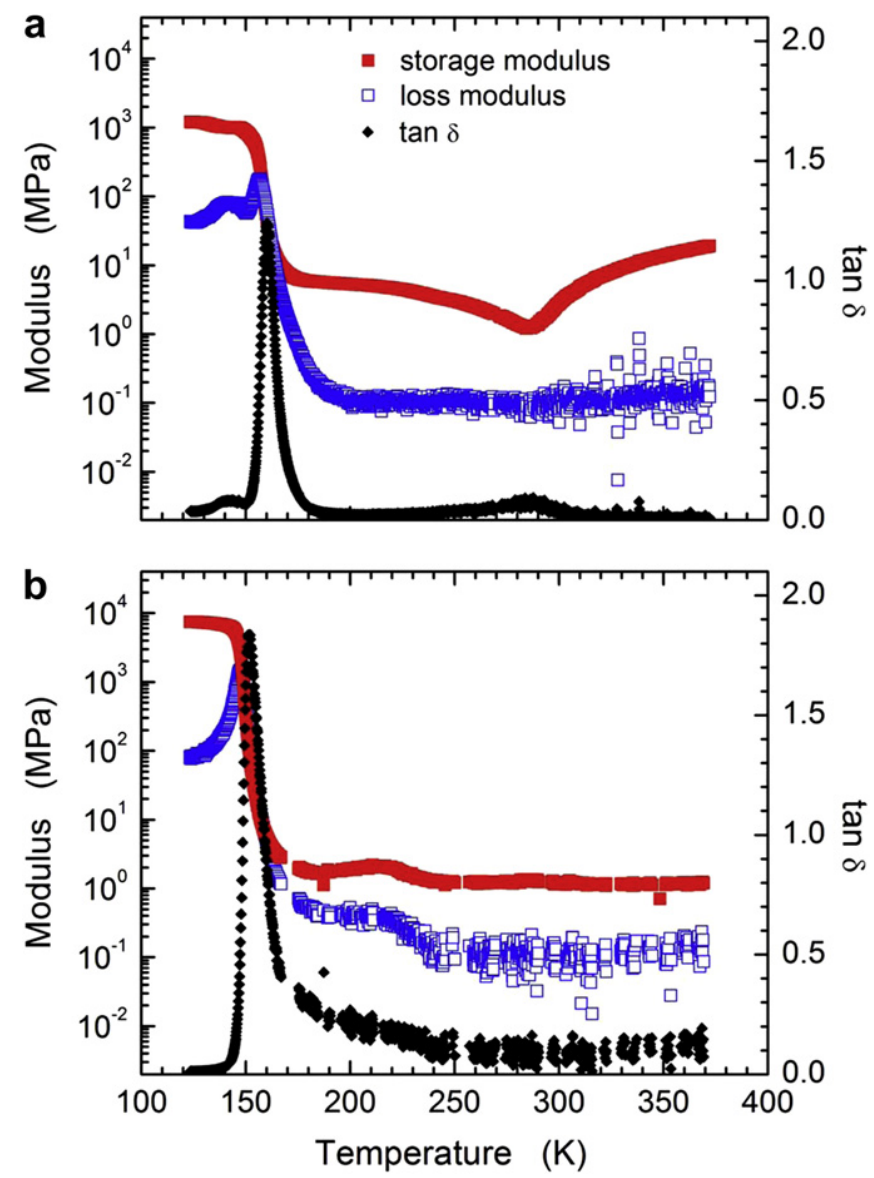

Fig. 2. Dynamical mechanical analysis data for 11-250 (a) and 11-75 (b) PDMS-PVMS formulations performed at $1 \mathrm{~Hz}$. Storage modulus ( $\square$ ) and loss modulus ( $\square$ ) (left ordinate) and $\tan \delta(\bullet)$ (right ordinate) as a function of temperature.

studied is that 11-75 does not exhibit the characteristic rise in $E^{\prime}$ at temperatures $>298 \mathrm{~K}$. This may be important in the design of surfaces for tissue-culture as incubation occurs typically at $\approx 310 \mathrm{~K}$. Assimilation of relevant work by other researchers reveals that most mechanical property data are recorded at room temperature; this could be a variable overlooked as the elastic modulus can vary with temperature. It would be prudent to explore two areas in future studies. These involve: i) discerning the differences between 11-75 and 11-250 thermoelastic behavior and ii) reevaluating the mechanical properties at incubation temperature for the polymers used in biomedical applications.

We assessed the surface properties of the PDMS-PVMS network films by measuring the contact angle of deionized water $\left(\theta_{\mathrm{DIW}}\right)$ via static contact angle method and the dynamic contact angle (DCA) measurement and compared them to the "benchmark" samples comprising PVMS and PDMS (Sylgard-184) networks. Recent work on PDMS networks reported on contamination of the probing liquid during dynamic contact angle measurements [20]. Given the very short exposure of our samples to deionized water, we do not believe any contamination occurs during the course of our experiments because the data we report below are fully consistent with values reported previously on extracted SE networks. The general trends observed support the notion that cross-linking leads to the loss of surface mobility of the SE chains in the network. In Fig. 3 we plot the advancing $\theta_{\text {DIW }}$ (a) and the rate of change of $\theta_{\text {DIW }}$ with time (b) as a function of the exposure time of the networks to DIW. In our studies the PDMS networks remained hydrophobic during the 

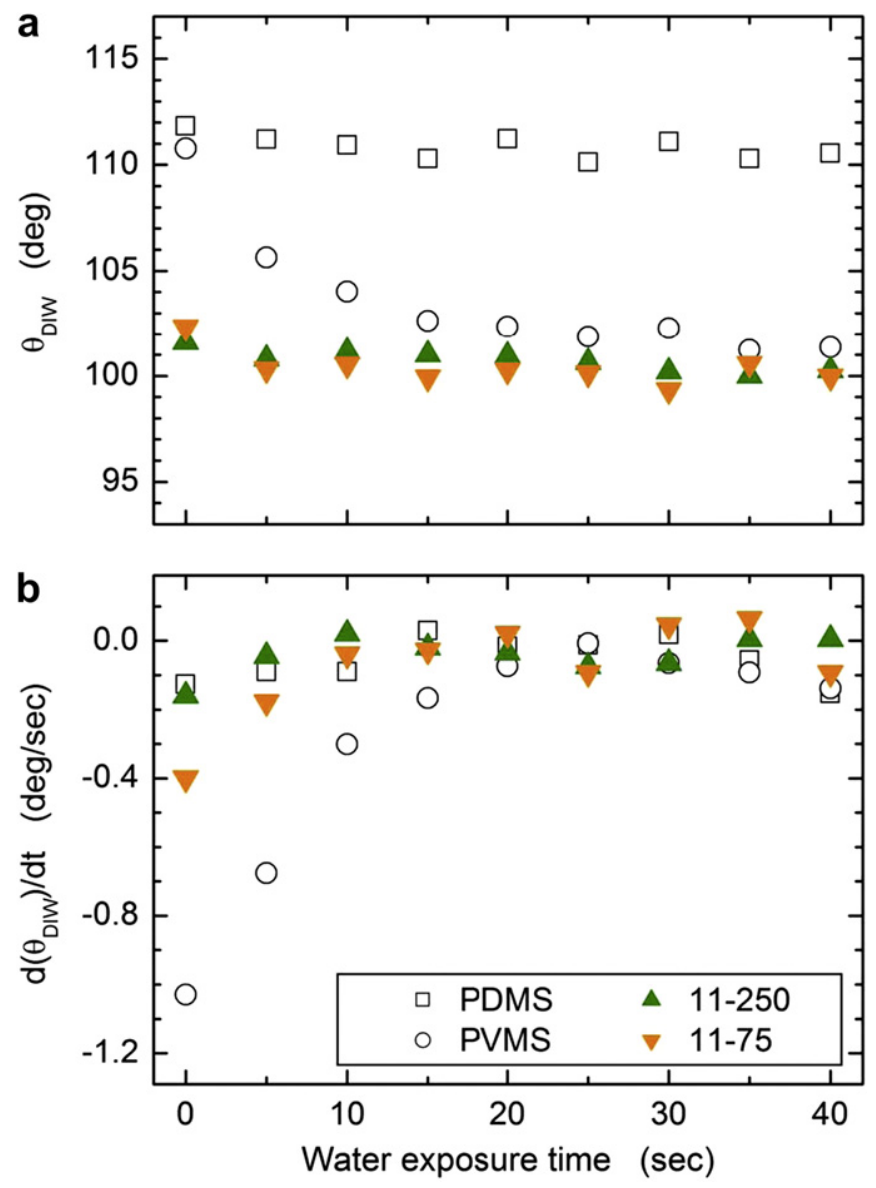

Fig. 3. (a) static contact angle and (b) wettability change using DI water for PVMS ( $O$ ), Sylgard-184 ( $\square)$, and PDMS-PVMS networks 11-250 ( $\mathbf{\Delta})$, and 11-75 ( $\nabla)$.

entire duration of the test. In contrast, PVMS exhibited the wellknown surface reconstruction [16]. The PDMS-PVMS network remained more hydrophilic relative to pure PDMS networks during the tests suggesting that a considerable amount of vinyls (being more hydrophilic than methyls) was present on the sample surface. While the static $\theta_{\text {DIW }}$ did not reveal any significant differences in the PDMS-PVMS networks relative to the pure component SE networks, more insight into the network structure could be gained by examining the water contact angle hysteresis $\left(\Delta \theta_{\text {DIw }}\right)$, i.e., the difference between the advancing and receding dynamic $\theta_{\text {DIW }}$ using the DCA ( $c f$. Fig. 56 in the Supplementary information). Generally, the data in Fig. 3 indicate that the surface reconstruction for $11-V_{\text {PVMS }}$ in the presence of water for is both slower and of lower magnitude than PVMS. This can be attributed to not only the composition change but possibly due to the reduction in $M_{\mathrm{C}}$ for $11-V_{\text {PVMS }}$ relative to PVMS. This reduction in $M_{C}$ may be reducing the backbone mobility and hinder the ability of the vinyl substituents to reconstruct at the surface. General assumptions of contact angle measurements assume the following properties of a surface: smooth, homogeneous, non-deformable, non-reacting, non-penetrable, with no reorientation, and zero mobility [21]. Siloxane surfaces do not fit these categories primarily due to surface reorientation; $\Delta \theta_{\text {DIW }}$ will typically increase with increasing the mobility of the functional groups on the surface [22,23]. Because molecular mobility is believed to affect greatly the adsorption of biological moieties on man-made surfaces [24], it may be worthwhile to explore the reversible characteristic in PDMS-PVMS networks in further detail in future studies.
Having characterized the properties of the PDMS-PVMS networks, we set to prepare modulus gradients by allowing equal amounts of two PDMS-PVMS formulations with a different content of the PDMS ad PVMS constituents to inter-diffuse and cure the materials in order to form networks with a position-dependent modulus. Two formulations, comprising PDMS-PVMS 15-30 and $15-125$ were poured into a $30 \times 30 \mathrm{~cm}^{2}$ polystyrene Petri dish in $30-\mathrm{g}$ amounts covering two adjacent and equal areas in the Petri dish. After curing for 3 -days in an oven at $70^{\circ} \mathrm{C}$, small sample pieces were taken along the substrate in the direction of the formed gradient in order to measure the storage modulus. We assumed that the modulus in each specimen (disks having $\approx 8 \mathrm{~mm}$ in diameter) was uniform. In Fig. 4 we plot the storage modulus as a function of the position on the sample using the solid squares. Adjacent samples were also taken from the substrate after an additional 5 days of curing at room temperature. This second set of data, depicted with open squares, exhibits values that are consistently higher in modulus than the earlier set indicating that additional cross-linking took place in the network during the 5-day period.

Overall, these results thus demonstrate that it is possible to prepare substrates comprising a gradient in modulus by interdiffusing two (or more) SEs and cross-linking them into the final network. They also point out some limitations of the current method. The first and most severe one is the inability to control the extent of the gradient in a precise manner. To that end, the spatial extent of the gradient is determined solely by the diffusivity of the two formulations and as such it will always be in the centimeter range. Additionally, because each formulation comprises a twocomponent mixture of PDMS-H and PVMS, the distribution of these two species in the final inter-diffused network may not be uniform that would ultimately lead to a certain "skewness" of the gradient that would be difficult to control. Finally, as documented above, the surface characteristics (i.e., the dynamics of the groups at the surface) of the individual formulations may vary to some extent, which will ultimately affect the application of such gradient structures in biological studies. We did not quantify the diffusion of the two components in the gradient set up quantitatively as this would involve determining the self-diffusion coefficients of both

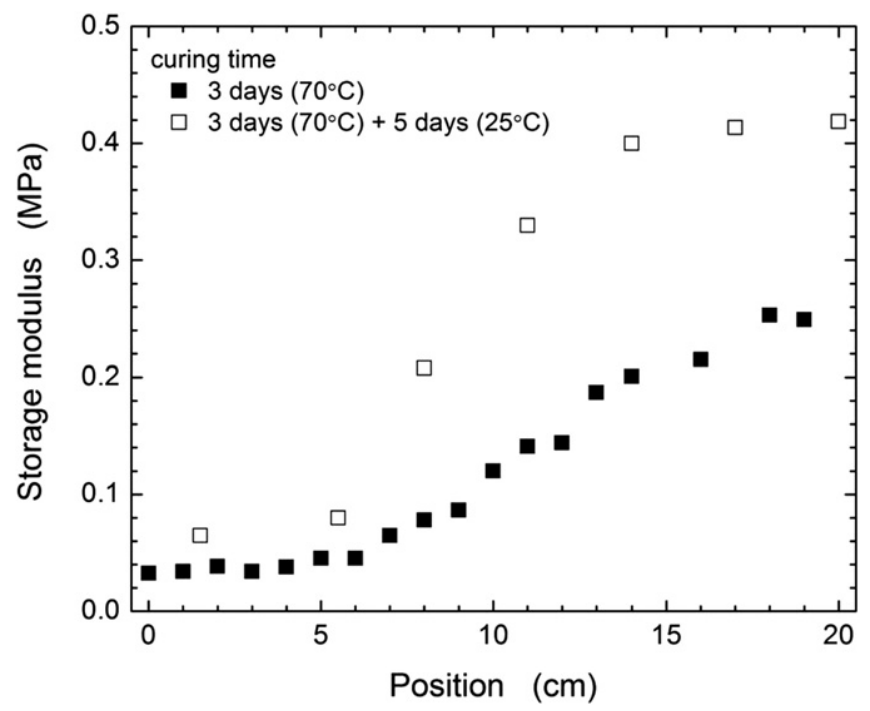

Fig. 4. Inter-diffusion of two formulations of PDMS-PVMS networks (15-30 and 15-125). The storage modulus was measured on an ARES 2000 rheometer at $1 \mathrm{rad} / \mathrm{s}$ within the linear viscoelastic regime. The first set of data ( $)$ was collected after a 3-day cure time in a $70{ }^{\circ} \mathrm{C}$ oven, the second set was collected after an additional 5-day room temperature cure time $(\square)$. The samples were taken from the same cured substrate. 
PVMS and PDMS as well as the mutual diffusion of the couple. In addition detailed information would be needed to quantify the diffusivity of the catalyst. Finally, deuterium-labeled components would have to be available so that one can achieve sufficient contrast between the two components for subsequent neutron or other analytical experiments. Those studies are beyond the scope of this paper. While we demonstrated the applicability of modulus gradient substrates in cell adhesion and proliferation studies [14], we attempted to develop a more robust and more tailored and general means of fabricating substrates comprising positiondependent variation of modulus. Those efforts are described in detail in the following section.

\subsection{Substrates with gradient in modulus based on UV-induced cross-linking}

In order to circumvent the obstructions associated with preparing substrates comprising gradient in modulus by interdiffusion of SE mixtures, we opted for generating such structures by UV-induced cross-linking of end-functionalized PVMS. As will be demonstrated below, the chief advantage of this technique is that it not only eliminates the aforementioned shortcomings on the interdiffusion method but it also enables one to prepare various motifs, shapes and "sharpness" of the modulus regions on the substrate. The basic principle of this new methodology is simple. We cast thin films of UV-curable SEs and set in the modulus by exposing them to UV light across a photolithographic mask. In a sense, this method is similar to the technique used by Wong and co-workers in preparing PAAm networks $[8,9]$.

Despite the wide range of available UV-cure mechanisms that have been implemented with SE precursors, commercial-grade UVcurable silicones were not suitable to our studies. These included, for instance, SEs manufactured by Loctite Corporation that utilized dual-curing mechanisms. The primary role of this elastomer class is to provide the construction industry with a rapid surface-cure via UV-induction in conjunction with a secondary (and slower) alkoxycure mechanism for the complete substrate cure. This precluded these elastomers for use in a gradient-cure mechanism, as they would eventually cure uniformly throughout the substrate with the secondary cure. While Gelest and Dow Corning provide UV-curable silicone formulations they are aimed for resin-grade coatings. Use of the commercial-grade elastomers would also limit surface modification as they are formulated with 100\% PDMS. Products by Dow Corning Corporation, General Electric Company, and Loctite Corporation dominate the majority of UV-curable siloxane synthesis work under various patent landscapes. Siloxane polymers have been formulated with UV-curable functionality such as acrylates, epoxides, norbornenes, and thiols [25-33]. Each mechanism possesses its own unique challenges. Due to the desire to utilize PVMS-OH fluid, we opted for a condensation reaction of PVMS-OH with mercaptoalkoxysilanes [34]. This seems to be a logical choice as: i) this method avoids the oxygen inhibition presented by acrylate functionality (of a concern since SEs are highly permeable to oxygen, ii) epoxide functionalization of PVMS-OH would prove challenging due to moisture inhibition, and iii) the desired norbornene intermediate would require additional synthesis steps. The thiol-ene reaction has recently regained popularity due to the excellent mechanical properties realized in the products, new synthesis routes to mask the odor characteristic of thiol monomers, low inhibition due to oxygen and moisture, and the development of new photoinitiators that allow for better oxidative stability (low-yellowing) [35]. Endcapping PVMS-OH with thiol functionality seems thus straightforward; it would lead to an SE system comparable to a PDMS analog that was reported to exhibit a rapid-cure and produced elastomers with superior mechanical properties [32].
The synthesis route used to prepare mercapto-terminated PVMS (PVMS-SH) SEs was adapted from the condensation process of linear dimethyl silanols ( $c f$. Scheme 2) [34]; see Experimental section for details. Qualitative analysis of the NMR spectra, molecular weight analysis, and successful UV-curing of the PVMSSH product (provided in the Supplementary information) validated our reaction scheme and process. The next step was to produce networks with the material cured via UV light. The PVMSSH possessed little odor and a photoinitiator 2-hydroxy-2-methyl1-phenyl-propan-1-one (Darocur ${ }^{\circledR}$ 1173), commonly used for biomedical applications, was soluble in PVMS. We explored two simple formulations for our networks: i) a blend of 65\% of PVMS$\mathrm{SH}(\approx 41 \mathrm{kDa})$ and $35 \%(\mathrm{w} / \mathrm{w})$ of PVMS-OH $(\approx 28 \mathrm{kDa})$ with $2-3 \%$ of photoinitiator and ii) $100 \%$ PVMS-SH ( $\approx 58 \mathrm{kDa}$ ) with $2-3 \%$ photoinitiator. The blend formulation was an attempt to incorporate PVMS with a lower polydispersity index into the mixture, reduce the amount of available cross-linking, and provide a broader distribution of cross-linked density throughout the cured elastomer. Scheme 2 depicts the general synthesis of a network with $100 \%$ of PVMS-SH polymer. The reaction proceeds with hydrogen abstraction from the sulfur atom after UV-initiation of the photoinitiator. The thiol radical attacks the vinyl substituent, whereas the formed carbon radical abstracts hydrogen from another mercapto- group. Cross-linking of the siloxane-ene and thiol-terminated siloxane continues until the radicals are terminated or UV-exposure is eliminated. As homopolymerization of the siloxane-ene component is not favored, only with prolonged UV-exposure will vinyl-to-vinyl cross-linking likely occur. This undesirable cross-linking will reduce the overall elasticity of the substrate. In the materials section we pointed out that additional increase of the modulus of the network may occur due to crosslinking of the unreacted methoxy groups present in the thiolbased siloxane. However, we do not anticipate this to take place at room temperature.
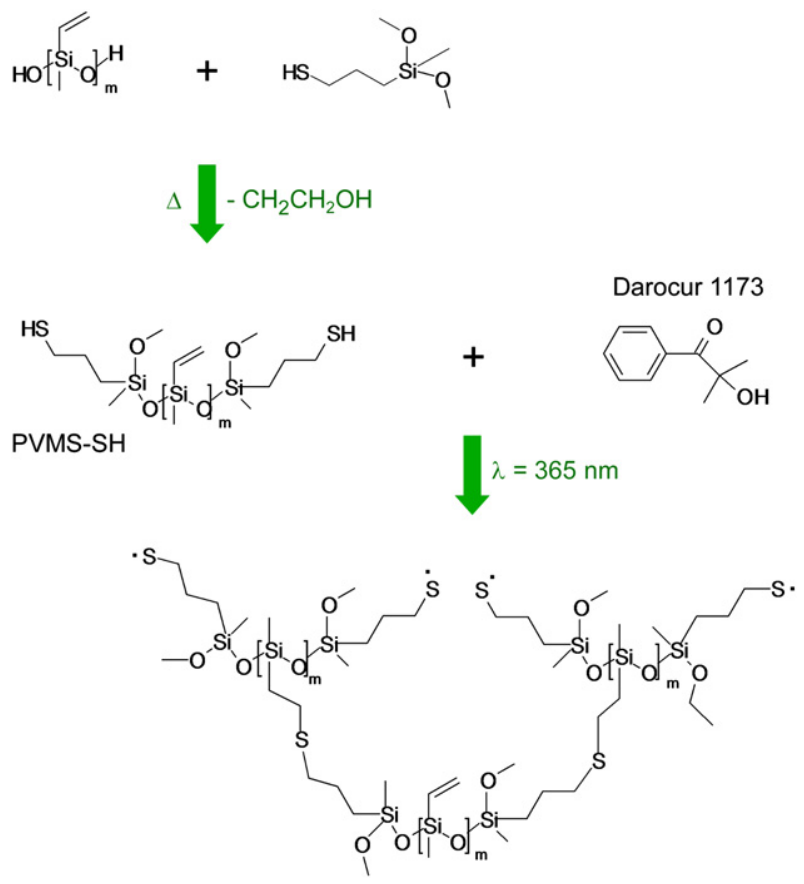

Scheme 2. Schematic for making mercapto-based poly(vinylmethylsiloxane) (PVMS) networks. The coupling reaction between 3-mercaptopropylmethyldimethoxy silane (MPMDMS) and PVMS was performed in reflux mode at $\approx 60^{\circ} \mathrm{C}$ for $\approx 2 \mathrm{~h}$. The SE network was generated by UV-assisted cross-linking of PVMS-MPMDMS at room temperature. 


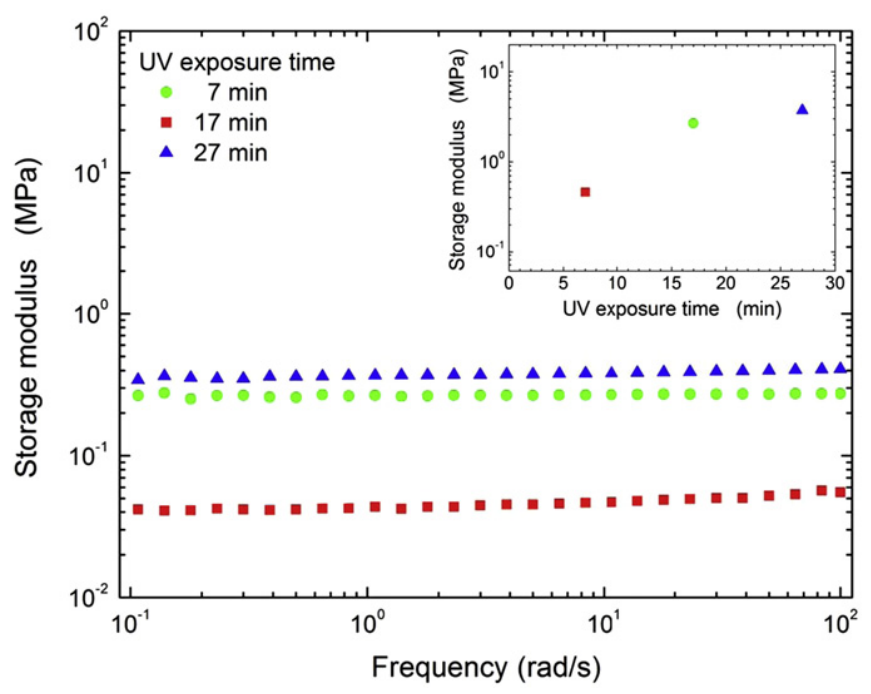

Fig. 5. Dynamic rheology frequency sweep data for a network of 65/35 PVMS-SH/ PVMS blend UV cured at $\lambda=365 \mathrm{~nm}$ for $7(\mathbb{0}), 17(\bullet)$, and $27(\boldsymbol{\Lambda})$ minutes. Scans performed at $30{ }^{\circ} \mathrm{C}$ and at low strain $(0.1 \%)$ determined to be within the LVE region.

Forming networks with 100\% PVMS-SH increases the likelihood that more SE chain segments are incorporated in the network. As the number of available cross-link sites greatly outnumbers the
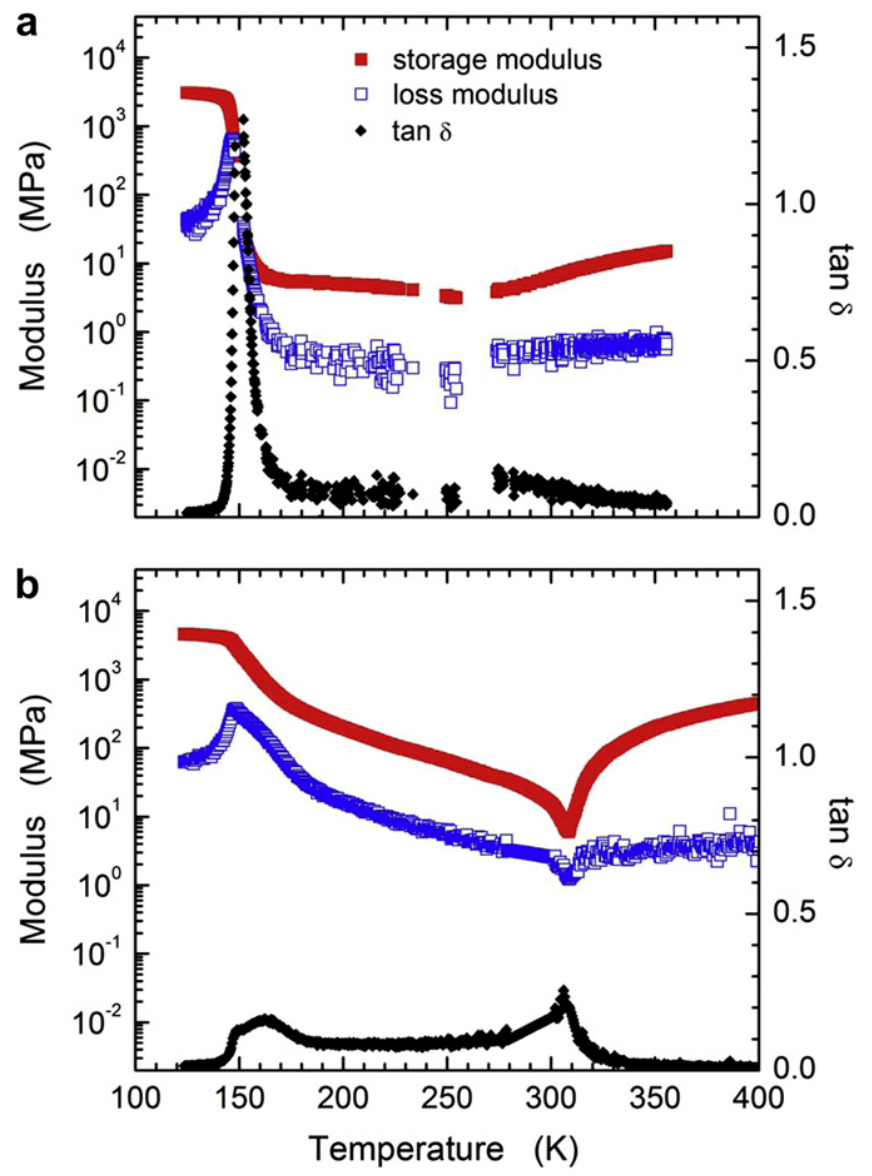

Fig. 6. Dynamic al mechanical analysis for PVMS cured via an alkoxy mechanism (a) and 100\% PVMS-SH UV-cross-linked material (b) performed at $1 \mathrm{~Hz}$. UV cure performed at $365 \mathrm{~nm}$ for $7 \mathrm{~min}$. Storage modulus ( $\square$ ) and loss modulus ( $\square$ ) (left ordinate) and $\tan \delta(\bullet)$ (right ordinate) as a function of temperature.
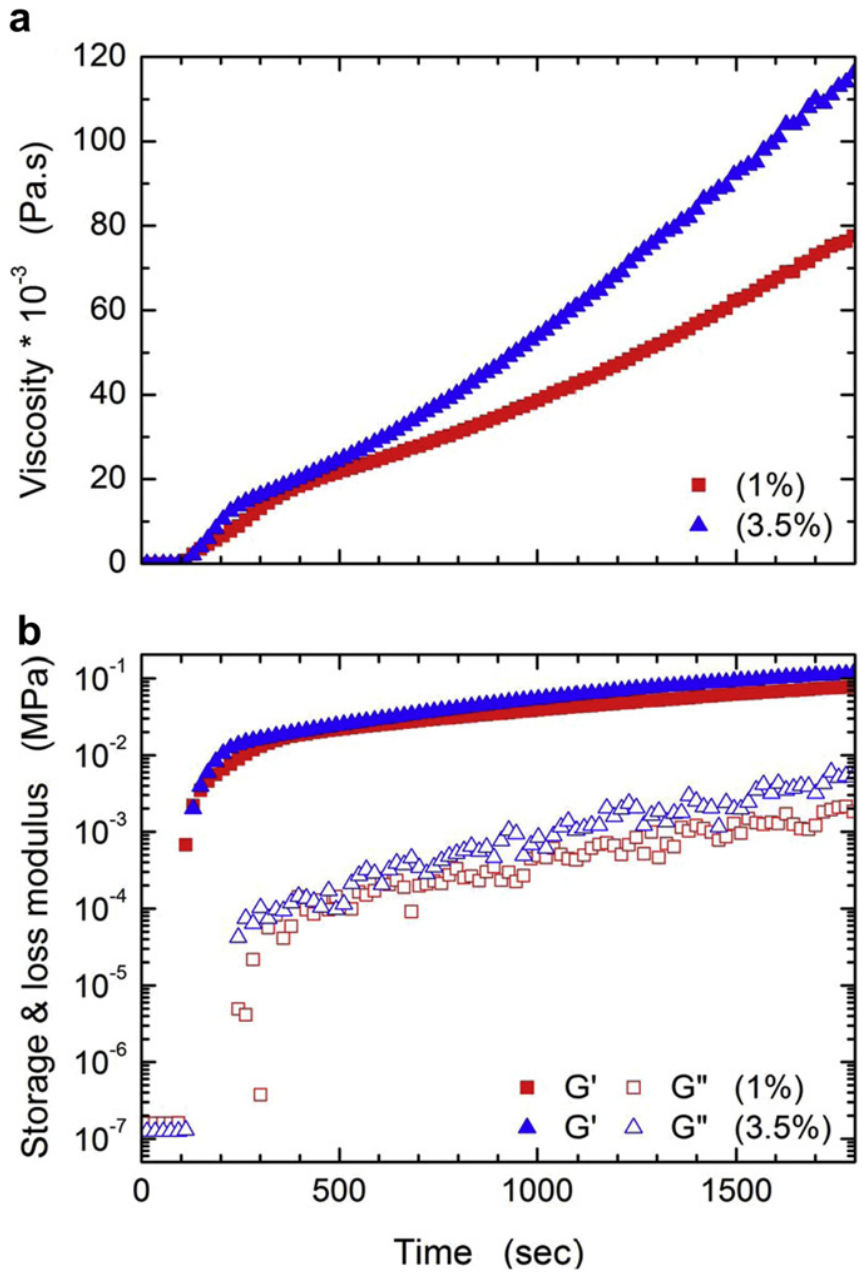

Fig. 7. Viscosity (a) and modulus (b) of PVMS-SH networks UV cured at $\lambda=365 \mathrm{~nm}$ for $2000 \mathrm{~s}$ having 1 (squares) and 3.5 (triangles) \% of the photoinitiator.

potential of thiol radicals generated, formulating with a blend of PVMS-SH and PVMS-OH reduces the total number of PVMS-S• available to react across the $\mathrm{C}=\mathrm{C}$ substituent of the siloxane backbone. This could result in some "dangling" PVMS chains in the network. With either formulation, if termination occurs between two PVMS-S• segments, it would act as a chain extender. A common concern regarding the formation of the $-\mathrm{Si}-\mathrm{C}-\mathrm{C}-\mathrm{S}-\mathrm{C}-$ bond is its thermal stability due to the predicted liability of heteroatomic bonds beta to the silicon atom [31]. For example, a significant reduction in weight loss at $360^{\circ} \mathrm{C}$ was reported in a PDMS-coPVMS copolymer modified with 2-(2-benzimidazolyl) ethanethiol compared to the weight loss at $500{ }^{\circ} \mathrm{C}$ with the unmodified PDMSco-PVMS [36]. While this thermal instability may preclude the use of SE thiol-ene elastomers at high temperatures, they are still suitable for current research where temperatures will remain below $100{ }^{\circ} \mathrm{C}$.

Rheological properties of UV-cured PVMS-SH networks were obtained with an ARES rheometer operating isothermally at $30^{\circ} \mathrm{C}$ within the linear viscoelastic regime for elastomers cured at 365 $\mathrm{nm}$ for various times (cf. Fig. 5). Increasing the exposure time from 7 to 27 min resulted in an order of magnitude change (from 30 to 300 $\mathrm{kPa})$ in the storage modulus $\left(G^{\prime}\right)$. This range in modulus showed promise for generating compliance gradients by simply varying UV dose by tailoring the time and/or intensity of the UV light exposure. A control sample for the $100 \%$ PVMS-SH formulation was analyzed 

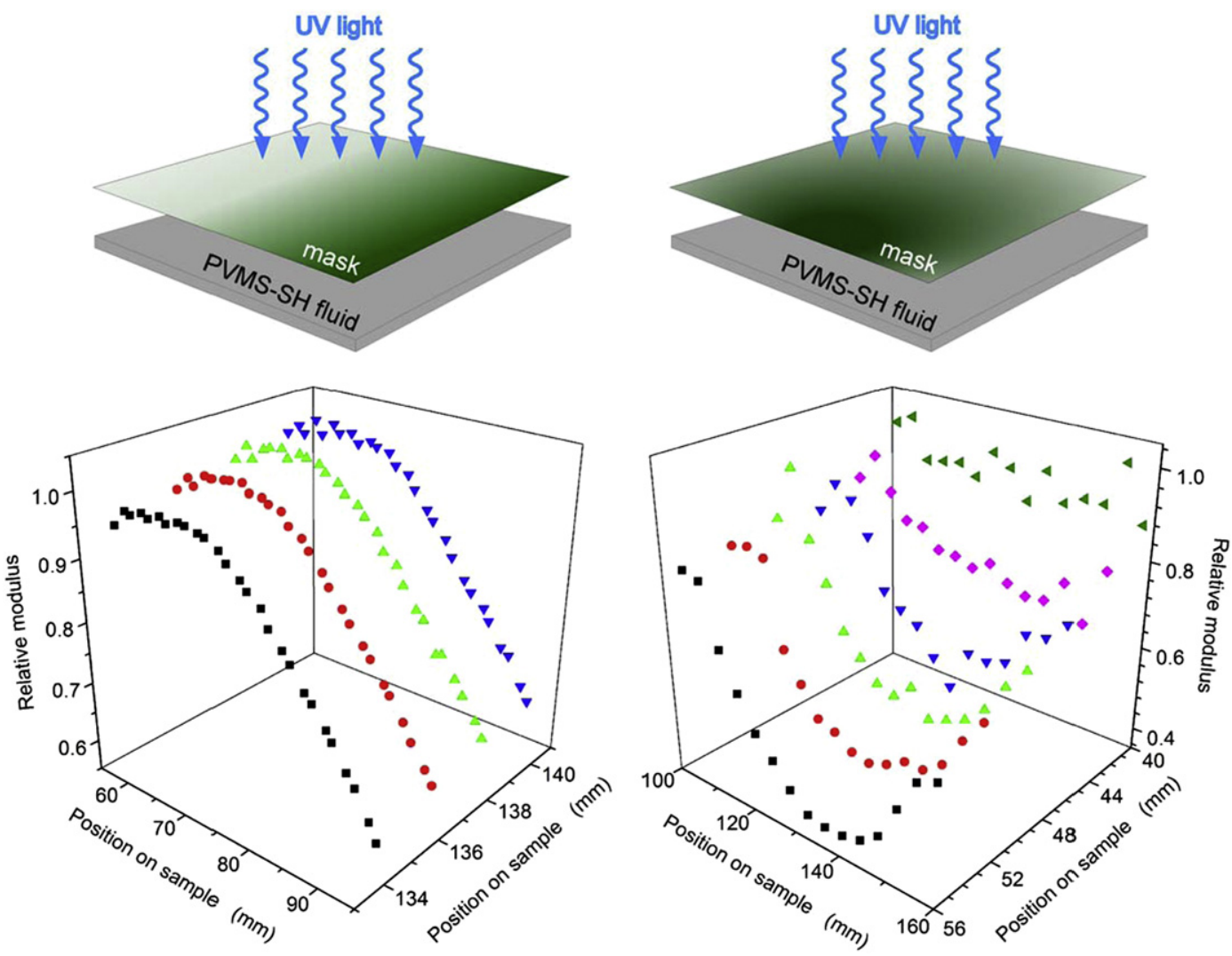

Fig. 8. Modulus of PVMS-SH networks UV cured across various masks. The modulus data were rescaled to the highest modulus value measured in each sample. Darocur 1173 was used as a photoinitiator at 3 weight $\%$ of the total formulation.

with DMTA in the temperature ramp mode. The glass transition temperature, determined by the large step change in storage modulus was $150 \mathrm{~K}$, similar to standard alkoxy-cure PVMS (cf. Fig. 6). Closer inspection of the DMTA data reveals a large disparity between the $\tan \delta$ for PVMS and PVMS-SH. With standard alkoxy-cure PVMS, the cross-linking occurs at the chain ends. This is not the case for PVMS-SH where backbone cross-linking is the mode for network formation. As $\tan \delta=E^{\prime \prime} / E^{\prime}$, it is apparent with the data for PVMS-SH that the elastic component is significantly higher for PVMS-SH in the rubbery regime as $\tan \delta$ approaches zero; indicative of a solid-like behavior. This result is logical as the backbone flexibility is significantly reduced through cross-linking of the vinyl substituents thus reducing the molecular weight (and motion) between cross-links. Although the modulus goes up as a function of temperature as seen in other siloxane networks [37], it increases at a much higher rate $\left(d E^{\prime} / d T=4.8 \mathrm{MPa} / \mathrm{K}\right.$ for PVMS-SH $v s$. $0.181 \mathrm{MPa} / \mathrm{K}$ for PVMS). In addition, this temperature-dependent increase in modulus occurs at $\approx 310 \mathrm{~K}$ for the PVMS-SH network $v$. $\approx 285 \mathrm{~K}$ for the PVMS network. Two phenomena could be influencing the increase in modulus with respect to temperature. The first could simply involve the generation of additional cross-linking points throughout the network. This was not the case with PVMS as post-testing after the temperature ramp did not indicate an increase in $E^{\prime}$. Post-testing was not performed with the PVMS-SH network which is necessary to determine if further cross-linking occurs at elevated temperatures. The other phenomenon would follow the theory of rubber elasticity where the elastic modulus is directly proportional to temperature as described by:

$E^{\prime}=3 \frac{\rho R T}{M_{\mathrm{C}}}$ where $\rho$ is the polymer density, $R$ the gas constant, and $M_{\mathrm{C}}$ the molecular weight between cross-links [38]. Equation (4) assumes that the overall polymer strand molecular weight is much larger than $M_{\mathrm{C}}$, which is the case for PVMS-SH networks but does not hold for alkoxy-cured PVMS networks, where the following relation would apply:

$E^{\prime}=3 \frac{\rho R T}{M_{\mathrm{C}}}\left(1-\frac{2 M_{\mathrm{C}}}{M_{\mathrm{n}}}\right)$

where $M_{\mathrm{n}}$ is the average polymer strand molecular weight [38]. As $M_{\mathrm{n}}$ approaches $M_{\mathrm{C}}$ this relation fails as the quantity $\left(1-2 M_{\mathrm{C}} / M_{\mathrm{n}}\right)$ becomes negative. Nonetheless, it is instructive to assess the degree of cross-linking for PVMS-SH networks in relation to alkoxy-cured PVMS networks. As can be expected due to the differing crosslinking methods, the slope of a linear fit of $E^{\prime} v s$. temperature is 30 times steeper for PVMS-SH than alkoxy-cured PVMS indicating increased cross-linking for PVMS-SH. Comparing $E^{\prime}$ at room temperature and at $310 \mathrm{~K}$ (cell incubation temperature) yields additional information worthy of further investigation. At $310 \mathrm{~K}$ the moduli of PVMS-SH and PVMS are very similar (8.24 and 6.31 MPa, respectively). At room temperature, the modulus of PVMS-SH is twice as much as PVMS. More dynamic rheology experiments performed isothermally would elucidate if these relative differences are accurate.

We have also attempted to monitor the formation of the SE networks in situ by UV-induced cross-linking of PVMS-SH materials via dynamic rheology (TA AR2000 rheometer). Fig. 7 depicts the viscosity and modulus of the SE network during the crosslinking process. UV light at a wavelength of $365 \mathrm{~nm}$ was directed at the formulated uncured PVMS-SH fluid (at two levels of the 
Darocur 1173 photoinitiator) between the parallel plate geometry. During the UV-exposure time, a dynamic time sweep was performed for each formulation at a stress of $50 \mathrm{~Pa}$ and a frequency of 1 $\mathrm{rad} / \mathrm{s}$. The rate of cross-linking is illustrated in the data shown in Fig. 7 by the sharp increase in modulus up to $200 \mathrm{~s}$ at which point the UV source is eliminated but the monitoring of the rheological properties continues. The elastic modulus $G^{\prime}$ rate of change during the exposure time was $62.7 \mathrm{~Pa} / \mathrm{s}$ for $1 \mathrm{wt} \%$ photoinitiator versus the faster rate of $98.6 \mathrm{~Pa} / \mathrm{s}$ for $3.5 \mathrm{wt} \% \mathrm{PI}$. After the UV-exposure time, the rate of cross-linking continues but at a reduced rate for both concentrations. We also note that while the 3.5\% cross-linker addition cures at a slightly faster rate, the $1 \%$ cross-linker formulation plateaus to an equivalent elastic modulus after $500 \mathrm{~s}$. To minimize residual cross-linker in the resultant SE, the lower addition amount is preferred for the formulating of SEs. Because we were unable to determine the intensity of the UV light inside the parallel plate in the rheometer, we cannot relate the absolute values of $G^{\prime}$ and $G^{\prime \prime}$ directly to the UV dose. While we cannot compare the rheometer-based modulus values directly to other data on UVcured elastomers, where the relationship between the UV dose and the modulus can be obtained ( $c f$. Fig. S7 in the Supplementary information), the trends in $G^{\prime}$ and $G^{\prime \prime}$ given in Fig. 7 are clear and demonstrate that networks are formed $\left(G^{\prime}>G^{\prime \prime}\right)$ and the modulus of the network increases with increasing UV dose.

In order to create substrates comprising a position-dependent modulus, glass slides were coated with the uncured PVMS-SH formulation, covered with a patterned transparency film bearing a predetermined transparency motif, and exposed to UV (cf. Fig. 8). After the desired exposure time (ranging from 5 to $20 \mathrm{~min}$ ), the film was removed from the UV source and placed in the dark to prevent subsequent cross-linking. Mechanical probing of the material modulus in the $\mathrm{X}-\mathrm{Y}$ plane was carried out using a Hysitron Triboindenter in displacement controlled indentation mode to probe the same depth for every indentation. A series of model studies was conducted that enabled linking the measured modulus of the SE network to the UV dosage, given by the product of the UV-exposure time and UV light intensity; the latter was assessed directly by determining the intensity of the UV light after passing it through masks with different transparencies. An example of such a calibration relationship is provided in Fig. S7 in the Supplementary information. In order to demonstrate the capability of the method, in Fig. 8 we provide only two illustrative examples of such spatiallytuned modulus gradients. For simplicity, we present the results for the modulus data in the $\mathrm{X}-\mathrm{Y}$ plane on a relative basis, i.e., scaled relative to the highest modulus measured in the sample. These results illustrate that that one can produce readily substrates with in-plane variable modulus ranging from 40 to $100 \%$ of the maximum obtained modulus; additional tuning can be provided by varying the transparency of the mask, the UV dosage and the molecular weight of the PVMS-SH polymer. Work is currently in progress that addresses the effect of the aforementioned parameters and provides detailed range of the modulus values that can be achieved by this method. In addition, modulus changes upon ageing of the specimens after formation is being assessed and will be reported in the future.

\section{Conclusion}

Substrates comprising compliance gradients can be of aid in various field of materials science and in biomaterials, where the understanding of cell growth, proliferation, and migration on synthetic materials is required. Two methods leading to such structures based on SE networks have been reported in this paper. The first technique utilizes inter-diffusion of two SE formulations leading to SE networks with position-dependent gradually-varying modulus. While straightforward to implement, this methodology offers limited control over the spatial extent of the gradient. A more controllable way to fabricate substrates comprising spatial gradients in modulus is to utilize UV-curable SE materials and imprint the desired spatial variation of mechanical modulus by external UV mask. In the latter case, the reaction time for a cured network is less than $30 \mathrm{~min}$ with a direct UV source of $365 \mathrm{~nm}$. While more work is needed to fine tune these methods of a particular application, i.e., studying the effect of substrate rigidity on cell signaling locomotion (i.e., durotaxis), spreading and proliferation, the principles assembly developed in this work should pave their way towards utilization of such unique material structures.

\section{Note added in proof}

During the typesetting of the article we became aware of a recent paper reporting on the formation of stiffness gradient by photochemical, position-dependent degradation of a hydrogel network and its subsequent utilization in cell differentiation studies [Kloxin AM, Benton JA, Anseth KS. Biomaterials 2010, 31; 1].

\section{Acknowledgement}

This work was supported through the Office of Naval Research, Grant no. N00014-08-1-0369, and the award from the Army Research Office, Grant no. W911NF-07-1-0090. Partial supplemental funding from the Army Research Office, Grant no. W911NF07-1-0090 is also greatly appreciated. We thank Dr. Hana Gracz for her assistance with the NMR measurements, Professor Russell Gorga for use of the DMTA rheometer and Professor Saad Khan for the use of the ARES dynamic rheometer.

\section{Appendix. Supplementary data}

The supplementary materials can be viewed at doi:10.1016/j. polymer.2009.11.070.

\section{References}

[1] Ohring M. Materials science of thin films: deposition and structure. San Diego, California: Academic Press; 2002.

[2] Chattopadhyay R. Surface wear: analysis, treatment, and prevention. Ohio: ASM International, Materials Park; 2001.

[3] Genzer J, Groenewold J. Soft Matter 2006;2:310 [and references therein]

[4] Brady RF, Singer IL. Biofouling 2000;15:73.

[5] Brady RF. Prog Org Coatings 2001;43:188.

[6] Discher DE, Janmey P, Wang Y. Science 2005;310:1139 [and references therein].

[7] Kong HJ, Liu J, Riddle K, Matsumoto T, Leach K, Mooney DJ. Nature Mater 2005;4:460.

[8] Wong J, Velasco A, Rajagopalan P, Pham Q. Langmuir 2003;19:1908.

[9] Zaari N, Rajagopalan P, Kim S, Engler A, Wong J. Advanced Materials 2004; $16: 2133$.

[10] Wong J, Leach J, Brown X. Surface Science 2004;570:119.

[11] Engler A, Richert L, Wong J, Picart C, Discher D. Surface Science 2004;570:142.

[12] Lo C-M, Wang MB, Dembo M, Wang Y-l. Biophysical Journal 2000;79:144.

[13] Colas A, Cutis J. In: Ratner B, Lemons JE, Hoffman AS, Schoen FJ, editors. Biomaterials science: an introduction to materials in medicine. Elsevier; 1996.

[14] Weiger MC, Fraley K, Crowe-Willoughby JA, Genzer J, Haugh JM. in preparation.

[15] Braun JL, Mark JE. Macromolecules 2002;35:5273.

[16] Efimenko K, Crowe JA, Manias E, Schwark DW, Fischer DA, Genzer J. Polymer 2005;46:9329.

[17] Korth K, Albert P, Klefer I. US Patent \#6995280 B2; 2006.

[18] ACD/HNMR Predictor \& ACD/CNMR Predictor. Toronto: ACD/Labs, http://www. acdlabs.com/products/spec_lab/predict_nmr/; 2007.

[19] Taylor R, Parbhoo B, Fillmore D. In: Smith AL, editor. The analytical chemistry of silicones. New York: John Wiley \& Sons; 1991. p. 347-420.

[20] Uilk JM, Mera AE, Fox RB, Wynne KJ. Macromolecules 2003;36:3689.

[21] Andrade J. Surface and interfacial aspects of biomedical polymers. New York: Plenum Press; 1985.

[22] Crowe JA, Genzer J. Journal of the American Chemical Society 2005;127:17610.

[23] Crowe-Willoughby JA, Genzer J. Advanced Functional Materials 2009;19:460. 
[24] Arifuzzaman S, Özçam AE, Efimenko K, Fischer DA, Genzer J. Biointerphases 2009;4:FA33.

[25] Crivello J, Lee J. ACS Symp. Series 417. In: Hoyle C, Kinstle J, editors. Radiation curing of polymeric materials. Washington D.C.: American Chemical Society; 1990. p. 398.

[26] Eckberg RP, Riding KD. ACS Symp. Series 417. In: Hoyle C, Kinstle J, editors. Radiation curing of polymeric materials. Washington D.C.: American Chemical Society; 1990. p. 382.

[27] Fouassier JP. Photoinitiation, photopolymerization, and photocuring. Munich: Hanser Publishers; 1995

[28] Gordan DJ, Ziemelis MJ. US patent \#4107390; 1978.

[29] Goudy WG, Keil JW. US patent \#3,380,196; 1968.
[30] Homan GR, Lee C-1. US patent \#4066603; 1978.

[31] Jacobine A, Nakos S. In: Peppas NA, editor. Radiation curing. New York: Plenum Press; 1992. p. 181.

[32] Lee C-l, Lutz M. US Patent \#4780486; 1986.

[33] Mine K, Nalto H, Yamakawa K. European Patent \#0 728799 A1; 1996.

[34] Rees S, Westall S, European Patent \#0 382 365; 1990.

[35] Hoyle CE, Lee TY, Roper T. Journal of Polymer Science: Polymer Chemistry 2004;42:5301.

[36] Persson JC, Jannasch P. Macromolecules 2005;38:3283.

[37] Crowe-WilloughbyJ.A, Genzer J. in preparation.

[38] Murayama T. Dynamic mechanical analysis of polymeric materials. Amsterdam: Elsevier Scientific Publishing Company; 1978. 